\title{
Generalised Connections and Curvature
}

\author{
Michael Kunzinger, Roland Steinbauer \\ Department of Mathematics, University of Vienna, \\ Strudlhofg. 4, A-1090 Wien, Austria \\ e-mail: michael.kunzinger@univie.ac.at \\ e-mail: roland.steinbauer@univie.ac.at \\ James A Vickers \\ School of Mathematics, University of Southampton, \\ Highfield, Southampton SO17 1BJ, United Kingdom \\ e-mail: J.A.Vickers@maths.soton.ac.uk
}

August 14, 2018

\begin{abstract}
The concept of generalised (in the sense of Colombeau) connection on a principal fibre bundle is introduced. This definition is then used to extend results concerning the geometry of principal fibre bundles to those that only have a generalised connection. Some applications to singular solutions of Yang-Mills theory are given.
\end{abstract}

\section{Introduction}

Recently the theory of Colombeau algebras of generalised functions [6] 7] has been applied to a number of areas of geometrical interest such as the Lie group analysis of partial differential equations (e.g., [20, 8]) and the study of singular spacetimes in general relativity (see [30] for a survey). In order to address these geometric issues in a satisfactory manner it was necessary to reformulate the theory of Colombeau algebras to ensure that it was diffeomorphism invariant. This was accomplished for the so-called full theory (in which one has a canonical embedding of Schwartz distributions) in [13, 15] using calculus in 'convenient' 18. infinite-dimensional vector spaces. An alternative approach is to work instead with the so-called special variant of the theory. Although this version does not posses a canonical embedding of the space of distributions it may still be used to model singularities in a non-linear setting and has the added advantage that it is manifestly diffeomorphism invariant. As a result there has been considerable use of the special algebra in addressing geometric problems. See for example chapters 4 and 5 of 14 for applications to symmetries of differential equations and to general relativity. 
In 22] the construction of generalised functions in the special algebra was extended to the theory of generalised sections of vector bundles. In particular this approach was used to define generalised vector fields, tensor fields and differential forms thus providing a foundation for a non-linear theory of distributional geometry. This work was extended in 23] to give a description of generalised (pseudo-)Riemannian geometry. In particular the definitions of generalised pseudo-Riemannian metric, generalised Levi-Civita connection and generalised curvature tensor in this setting were given.

The aim of the present paper is to give a description of an equally important area of generalised differential geometry, namely the generalised theory of connections on principal fibre bundles and on associated vector bundles. Such a theory turns out to be important mathematically in the description of characteristic currents for singular connections and Chern-Weil theory for bundle maps [16 (see also 25]). In [16] Harvey and Lawson consider connections on a vector bundle $E$ over a manifold $M$ which are smooth over $M \backslash \Sigma$ where $\Sigma$ is a closed measure zero subset of $M$ called the singular set. They call such a connection a 'singular connection' and in order to compute its Chern currents the singular connection $\nabla$ is approximated by a family of smooth connections $\nabla_{\varepsilon}$, defined over the whole of $M$, which converge to $\nabla$ on $M \backslash \Sigma$ as $\varepsilon \rightarrow 0$. The problem with such an approach is that there are very many different ways of representing $\nabla$ in terms of a family of smooth functions and it is not always clear how the results depend on the particular choice. It is precisely this kind of issue that the Colombeau approach is able to examine using the concepts of equivalence and association. Singular connections also naturally arise in physics through singular Yang-Mills fields. The first example of a singular Yang-Mills field was the 'fractionally charged instanton' described by Forgasc et. al. 11. This is a (Euclidean) self-dual Yang-Mills connection on the 4-sphere with a singularity along a 2-sphere. In fact this solution is invariant under a circle action so as shown by Atiyah [1] can be viewed as a monopole on hyperbolic 3-space. These singular instanton solutions arise because of the existence of a locally flat connection with non-trivial holonomy associated with parallel transport around the singular 2-sphere and are very similar to the conical singularities studied in [5]

The plan of the paper is as follows. In section 2 we recall some basic facts about the description of both generalised functions and generalised sections of vector bundles in the special Colombeau algebra. In section 3 we recall the classical theory of connections, in section 4 we show how this can be extended to the generalised case and in section 5 we introduce the concept of the generalised curvature of a connection. Section 6 recalls the concepts of horizontal lift and holonomy in the classical case and shows how the theory of generalised functions taking values in a manifold [19, 24] and of generalised flows [21] enables these concepts to be extended to the generalised case. Section 7 introduces the notion of generalised connection on an associated vector bundle and the corresponding generalised covariant derivative and demonstrates that in the case of a generalised linear connection on $T M$ (where $T M$ is regarded as an associated vector bundle of the frame bundle $L M$ ) this precisely reproduces the definition given 
in 23]. Section 8 extends the notion of characteristic class to the generalised case and in section 9 we end by showing how weakly singular Yang-Mills connections (used for example to describe the fractionally charged instanton) may be represented by generalised connections on the whole space.

\section{Nonlinear distributional geometry}

To begin with, let us fix some notation from differential geometry and briefly recall the construction of generalised functions in the special Colombeau algebra. Our principal reference for notation and terminology from the theory of algebras of generalized functions is [14.

In what follows, $M$ will always denote a paracompact, smooth Hausdorff manifold of dimension $m$ and we denote vector bundles with base space $M$ by $(E, M, \pi)$ or by $E \rightarrow M$, for short. The space of smooth sections of a vector bundle $E \rightarrow M$ is denoted by $\Gamma(M, E)$. The $(r, s)$-tensor bundle over $M$ will be written as $T_{s}^{r}(M)$ and we denote spaces of tensor fields by $\mathcal{T}_{s}^{r}(M):=$ $\Gamma\left(M, T_{s}^{r}(M)\right), \mathfrak{X}(M):=\Gamma(M, T M)$ and $\mathfrak{X}^{*}(M):=\Gamma\left(M, T^{*} M\right)$, where $T M$ and $T^{*} M$ denote the tangent and cotangent bundle of $M$, respectively, while $\Omega^{r}(M):=\Gamma\left(M, \Lambda^{r}(M)\right)$ is the space of r-forms. For a section $s \in \Gamma(M, E)$ we call $s_{\alpha}^{i}=\Psi_{\alpha}^{i} \circ s \circ \psi_{\alpha}^{-1}$ its $i$-th component $\left(1 \leqslant i \leqslant n^{\prime}\right.$, with $n^{\prime}$ the dimension of the fibers) with respect to the vector bundle chart $\left(V_{\alpha}, \Psi_{\alpha}\right)$ over the chart $\psi_{\alpha}$.

The (special) algebra of Colombeau generalised functions on $M($ (9, 22]) is defined as the quotient algebra $\mathcal{G}(M):=\mathcal{E}_{M}(M) / \mathcal{N}(M)$ of the algebra $\mathcal{E}_{M}(M)$ of nets of smooth functions $\left(u_{\varepsilon}\right)_{\varepsilon \in(0,1]} \in \mathcal{C}^{\infty}(M)^{(0,1]}=: \mathcal{E}(M)$ of moderate growth modulo the ideal $\mathcal{N}(M)$ of negligible nets, defined, respectively, by the following asymptotic estimates

$$
\begin{aligned}
\mathcal{E}_{M}(M):=\left\{\left(u_{\varepsilon}\right)_{\varepsilon} \in \mathcal{E}(M): \forall K \subset \subset M, \forall P \in \mathcal{P}(M) \exists N \in \mathbb{N}:\right. & \\
& \left.\sup _{p \in K}\left|P u_{\varepsilon}(p)\right|=O\left(\varepsilon^{-N}\right)\right\} \\
\mathcal{N}(M):=\left\{\left(u_{\varepsilon}\right)_{\varepsilon} \in \mathcal{E}_{M}(M): \forall K \subset \subset M, \forall m \in \mathbb{N}_{0}:\right. & \left.\left.\sup _{p \in K}\left|u_{\varepsilon}(p)\right|=O\left(\varepsilon^{m}\right)\right)\right\},
\end{aligned}
$$

Here $\mathcal{P}(M)$ is the space of linear differential operators on $M$. Elements of $\mathcal{G}(M)$ are denoted by $u=\left[\left(u_{\varepsilon}\right)_{\varepsilon}\right]=\left(u_{\varepsilon}\right)_{\varepsilon}+\mathcal{N}(M)$. $\mathcal{G}(-)$ is a fine sheaf of differential algebras with the operation of Lie derivative (w.r.t. smooth vector fields) defined by $L_{\xi} u:=\left[\left(L_{\xi} u_{\varepsilon}\right)_{\varepsilon}\right]$.

$\mathcal{C}^{\infty}(M)$ is embedded into $\mathcal{G}$ by the "constant" embedding $\sigma$, i.e., $\sigma(f):=$ $\left[(f)_{\varepsilon}\right]$, rendering $\mathcal{C}^{\infty}(M)$ a subalgebra of $\mathcal{G}(M)$. There exist injective sheaf morphisms $\iota: \mathcal{D}^{\prime}\left({ }_{-}\right) \hookrightarrow \mathcal{G}\left({ }_{-}\right)$embedding the space $\mathcal{D}^{\prime}$ of Schwartz distributions into $\mathcal{G}$ and which coincide with $\sigma$ on $\mathcal{C}^{\infty}\left({ }_{-}\right)$(22] Th. 2).

The notion of association is employed to achieve compatibility with the respective distributional concepts. A generalised function $u=\left[\left(u_{\varepsilon}\right)_{\varepsilon}\right]$ is called associated with $0, u \approx 0$, if $\int_{M} u_{\varepsilon} \mu \rightarrow 0(\varepsilon \rightarrow 0)$ for all compactly supported onedensities $\mu$ and one (hence every) representative $\left(u_{\varepsilon}\right)_{\varepsilon}$ of $u$. If $\int_{M} u_{\varepsilon} \mu \rightarrow\langle w, \mu\rangle$ 
for some $w \in \mathcal{D}^{\prime}(M)$ then $w$ is called the distributional shadow (or macroscopic aspect) of $u$ and we write $u \approx w$.

Contrary to the distributional setting, there exists a characterization of Colombeau generalized functions by their point values on generalized points (this property may be viewed as a nonstandard aspect of the theory). In fact, by inserting $p \in M$ into $u \in \mathcal{G}(M)$ one obtains a well-defined element of $\mathcal{R}$, defined as the set of moderate nets of numbers $\left(\left(r_{\varepsilon}\right)_{\varepsilon} \in \mathbb{R}^{(0,1]}\right.$ with $\left|r_{\varepsilon}\right|=O\left(\varepsilon^{-N}\right)$ for some $N)$ modulo negligible nets $\left(\left|r_{\varepsilon}\right|=O\left(\varepsilon^{m}\right)\right.$ for each $\left.m\right)$. More generally, $p \in M$ may be replaced by a generalized point $\tilde{p}$ and $u, v \in \mathcal{G}(M)$ coincide if and only if they attain the same point value on each $\tilde{p}$. For definitions and details we refer to [26, 22].

$\Gamma_{\mathcal{G}}(M, E)$, the $\mathcal{G}(M)$-module of generalised sections of a vector bundle $E \rightarrow$ $M$ is defined in a manner similar to that of generalised functions by using analogous asymptotic estimates with respect to the norm induced by any Riemannian metric on the respective fibres. Indeed, setting $\Gamma_{\mathcal{E}}(M, E):=(\Gamma(M, E))^{(0,1]}$ and letting $\mathcal{P}(M, E)$ denote the space of differential operators $\Gamma(M, E) \rightarrow \Gamma(M, E)$, we may define $\Gamma_{\mathcal{G}}(M, E):=\Gamma_{\mathcal{E}_{M}}(M, E) / \Gamma_{\mathcal{N}}(M, E)$, where

$$
\begin{aligned}
\Gamma_{\mathcal{E}_{M}}(M, E):=\left\{\left(s_{\varepsilon}\right)_{\varepsilon} \in \Gamma_{\mathcal{E}}(M, E):\right. & \forall P \in \mathcal{P}(M, E) \forall K \subset \subset M \exists N \in \mathbb{N}: \\
& \left.\sup _{p \in K}\left\|P u_{\varepsilon}(p)\right\|=O\left(\varepsilon^{-N}\right)\right\} \\
\Gamma_{\mathcal{N}}(M, E):=\left\{\left(s_{\varepsilon}\right)_{\varepsilon} \in \Gamma_{\mathcal{E}_{M}}(M, E):\right. & \forall K \subset \subset M \forall m \in \mathbb{N}: \\
& \left.\sup _{p \in K}\left\|u_{\varepsilon}(p)\right\|=O\left(\varepsilon^{m}\right)\right\}
\end{aligned}
$$

For the present purposes the bundles that we are most interested in are the generalised tensor bundles $\Gamma_{\mathcal{G}}\left(M, T_{s}^{r}(M)\right)$ which we denote $\mathcal{G}_{s}^{r}(M)$ and the generalised differential r-forms $\Gamma_{\mathcal{G}}\left(M, \Lambda^{r}(M)\right)$ which we denote $\Omega_{\mathcal{G}}^{r}(M)$. Generalised sections are denoted by $s=\left[\left(s_{\varepsilon}\right)_{\varepsilon}\right]=\left(s_{\varepsilon}\right)_{\varepsilon}+\mathcal{N}(M, E)$.

Again, smooth sections of $E \rightarrow M$ are embedded as constant nets, i.e., via $\Sigma: \Gamma(M, E) \hookrightarrow \Gamma_{\mathcal{G}}(M, E)$ by $\Sigma(s)=\left[(s)_{\varepsilon}\right]$ and we will usually suppress notationally the embedding $\Sigma$.

$\Gamma_{\mathcal{G}}(-, E)$ is a fine sheaf of $\mathcal{G}(M)$-modules and the $\mathcal{G}(M)$-module $\mathcal{G}(M, E)$ is projective and finitely generated (22, Th. 5). As $\mathcal{C}^{\infty}(M) \subset \mathcal{G}(M), \Gamma_{\mathcal{G}}(M, E)$ may alternatively be viewed as $\mathcal{C}^{\infty}(M)$-module and the two respective module structures are compatible with respect to the embeddings. [22], Th. 4 provides the following algebraic characterisation:

$$
\Gamma_{\mathcal{G}}(M, E)=\mathcal{G}(M) \otimes_{\mathcal{C}^{\infty}(M)} \Gamma(M, E),
$$

Generalised tensor fields may also be viewed as $\mathcal{C}^{\infty}(M)$-multilinear mappings taking smooth vector fields resp. one-forms to $\mathcal{G}(M)$ or as $\mathcal{G}(M)$-multilinear mappings taking generalised vector resp. covector fields to generalised functions, i.e., as $\mathcal{C}^{\infty}(M)$ - resp. $\mathcal{G}(M)$-modules we have (22], Th. 6)

$$
\begin{aligned}
& \mathcal{G}_{s}^{r}(M) \cong L_{\mathcal{C}^{\infty}(M)}\left(\mathfrak{X}^{*}(M)^{r}, \mathfrak{X}(M)^{s} ; \mathcal{G}(M)\right) \\
& \mathcal{G}_{s}^{r}(M) \cong L_{\mathcal{G}(M)}\left(\mathcal{G}_{1}^{0}(M)^{r}, \mathcal{G}_{0}^{1}(M)^{s} ; \mathcal{G}(M)\right) .
\end{aligned}
$$


The components of a generalised tensor field $T \in \mathcal{G}_{s}^{r}(M)$ with respect to the chart $\left(V_{\alpha}, \psi_{\alpha}\right)$ are given as the elements

$$
T_{\gamma_{1} \ldots \gamma_{s}}^{\alpha \beta_{1} \ldots \beta_{r}}:=\left.T\right|_{V_{\alpha}}\left(d x^{\beta_{1}}, \ldots, d x^{\beta_{r}}, \partial_{\gamma_{1}}, \ldots, \partial_{\gamma_{s}}\right)
$$

of $\mathcal{G}\left(V_{\alpha}\right)$. As before, compatibility with the purely distributional picture is accomplished via the notion of association and distributional shadow, cf. [22], Sec. 7 .

We refer to 22, 23, 14, for an introduction to further concepts of nonlinear distributional geometry in the Colombeau setting. In particular, in section 6 we will make use of the space $\mathcal{G}[M, N]$ of Colombeau generalized functions defined on the manifold $M$ and taking values in the manifold $N$. For definitions and properties of this space see [19, 23, 24].

\section{The Classical Theory of Connections}

Before developing the concept of a generalised connection we briefly review the classical theory of connections on a principal fibre bundle following the approach and (unless stated otherwise explicitly) notation of Kobayashi and Nomizu [17.

Let $P(M, G)$ be a principal fibre bundle over the manifold $M$ with structure group $G$ and projection $\pi: P \rightarrow M$. Given $x \in M$ then $\pi^{-1}(x)$ is a closed submanifold of $P$ which is diffeomorphic to $G$ and is called the fibre at $x$. If $p \in P$ and $X \in T_{p} P$ we say that $X$ is a vertical vector if it is tangent to the fibre through $p$. The space of vertical vectors at $p$ is denoted $V_{p}$ and is given by $V_{p}=\left\{X \in T_{p} P: \pi_{*}(X)=0\right\}$.

We now show how the space of vertical vectors at $p$ is isomorphic to the Lie algebra $\mathfrak{g}$ of $G$. The group $G$ acts freely on $P$ on the right

$$
\begin{aligned}
R: P \times G & \rightarrow P \\
(u, g) & \rightarrow u g=R_{g} u .
\end{aligned}
$$

Let $A \in \mathfrak{g}$ be an element of the Lie algebra of $G$, then $g_{t}=\exp (t A)$ is a 1-parameter subgroup of $G$ which generates a 1-parameter family of diffeomorphisms $\phi_{t}=R_{g_{t}}$ of $P$. Since the group action moves points along a fibre, the tangent to the orbit is a vector field $A^{*}$ which is also tangent to the fibre and is therefore a vertical vector field. We call $A^{*}$ the fundamental vector field corresponding to $A$ and denote the map from $A$ to $A^{*}$ by $\sigma$,

$$
\begin{aligned}
\sigma: & \mathfrak{g} \rightarrow \mathfrak{X}(P) \\
& A \rightarrow A^{*} .
\end{aligned}
$$

The above map is a Lie algebra homomorphism so that $\sigma([A, B])=[\sigma(A), \sigma(B)]$ where [,] denotes the bracket in the Lie algebra $\mathfrak{g}$ on the LHS and the Lie bracket between vector fields on the RHS.

If one restricts the map to a point $p \in P$ then

$$
\begin{aligned}
\sigma_{p}: & \mathfrak{g} \rightarrow V_{p} \\
A & \rightarrow A^{*}(p) .
\end{aligned}
$$


is a linear isomorphism between the Lie algebra and the space of vertical vectors at $p$.

We will also require the fact that the right action of $G$ on $P$ induces a corresponding action on the fundamental vector fields which satisfies the condition

$$
R_{g *}(\sigma(A))=\sigma\left(\operatorname{ad}\left(g^{-1}\right) A\right)
$$

where ad denotes the adjoint representation, ad : $G \rightarrow L(\mathfrak{g}, \mathfrak{g})$, of $G$ on $\mathfrak{g}$.

We are now in a position to make the following definition.

Definition 3.1 A connection form $\omega$ is a smooth 1-form on $P$ with values in the Lie algebra $\mathfrak{g}$ which satisfies the following conditions

(i) $\forall V \in V_{p}, \omega_{p}(V)=\sigma_{p}^{-1}(V)$

(ii) $\forall V \in T_{R_{g} p} P, \omega_{R_{g} p}(V)=\operatorname{ad}\left(g^{-1}\right) \omega_{p}\left(R_{g *}^{-1} V\right)$

Given a connection 1-form $\omega$ we may define $H_{p}$, the horizontal subspace of $T_{p} P$, to be the set of vectors annihilated by $\omega$, so that $H_{p}=\left\{X \in T_{p} P: \omega(X)=0\right\}$. Since $\omega_{p} \circ \sigma_{p}=\mathrm{id}$, the null space of $\omega_{p}: T_{p} P \rightarrow \mathfrak{g}$ is a $\operatorname{dim} M$ dimensional vector space transverse to the fibre. This allows one to uniquely decompose any vector into a vertical and horizontal part and to define projections $\pi_{v}: T_{p} P \rightarrow V_{p}$ and $\pi_{h}: T_{p} P \rightarrow H_{p}$. (Note that $\pi_{v}$ depends upon $\omega$ even though the space $V_{p}$ does not.) Furthermore (5) and condition (i) above ensure that condition (ii) holds automatically for vertical vectors. Thus condition (ii) amounts to the requirement that $R_{g *}$ takes horizontal vectors to horizontal vectors. This leads to the following alternative definition of a connection.

Definition $3.2 A$ connection on $P$ is an assignment of subspaces $H_{p}$ of $T_{p} P$ such that $p \mapsto H_{p}$ is a smooth distribution and which satisfies

(i) $T_{p} P=V_{p} \oplus H_{p}$

(ii) $H_{R_{g} p}=R_{g *} H_{p}$

\section{The Theory of Generalised Connections}

In order to define a generalised connection we replace the classical connection 1form with a generalised 1-form. As we saw in the previous section a connection form $\omega$ is a 1 -form on $P$ with values in the Lie algebra $\mathfrak{g}$. That is

$$
\omega \in \Omega^{1}(P, \mathfrak{g})=\Omega^{1}(P) \otimes_{\mathbb{R}} \mathfrak{g} .
$$

We therefore define a generalised connection 1-form to be an element of the space

$$
\Omega_{\mathcal{G}}^{1}(P, \mathfrak{g})=\Omega_{\mathcal{G}}^{1}(P) \otimes_{\mathbb{R}} \mathfrak{g} .
$$

We now turn to the conditions which ensure that the generalised form defines a connection. 
For a classical connection the first condition is that

$$
\omega_{p}(V)=\sigma_{p}^{-1}(V) \in \mathfrak{g}, \forall V \in V_{p} \subset T_{p} P
$$

or equivalently that

$$
\omega_{p}\left(\sigma_{p}\left(V^{*}\right)\right)=V^{*}, \forall V^{*} \in \mathfrak{g}
$$

For the case of a generalised connection we impose this requirement by demanding that the map $\omega_{p} \circ \sigma_{p}$ is the identity in the appropriate space. More precisely we require

$$
\left(p \mapsto \omega_{p}\left(\sigma_{p}(\cdot)\right)\right)=\mathrm{id} \in \mathcal{G}(P, L(\mathfrak{g}, \mathfrak{g})) .
$$

We now turn to the second condition which classically takes the form

$$
\omega_{R_{g} p}(V)=\operatorname{ad}\left(g^{-1}\right) \omega_{p}\left(R_{g *}^{-1} V\right), \forall V \in T_{R_{g} p} P
$$

It is less clear how this equation should be interpreted in the sense of generalised functions. To see this we consider the equivalent equation at the level of representatives of the generalised connection 1-form

$$
\omega_{R_{g} p, \varepsilon}(V)=\operatorname{ad}\left(g^{-1}\right) \omega_{p, \varepsilon}\left(R_{g *}^{-1} V\right)+N_{\varepsilon}
$$

where $N_{\varepsilon}$ is a term which is negligible. The difficulty comes from the fact that the other terms in the equation depend upon $g$ as well as $p, \varepsilon$ and $V$, so that we cannot simply take $N_{\varepsilon}$ to be an element of $\Omega_{\mathcal{N}}^{1}(P, \mathfrak{g}):=\Gamma_{\mathcal{N}}\left(P, T^{*} P\right) \otimes_{\mathbb{R}} \mathfrak{g}$ but we must also consider how $N_{\varepsilon}$ depends upon $g$. The attitude we will take is to regard $g$ as a parameter and therefore require $N_{\varepsilon}$ to satisfy the same estimates as an element of $\Omega_{\mathcal{N}}^{1}$ for any fixed value $g_{0}$ of $g$, or more generally for $g$ in some compact subset $L$ of $G$.

More precisely we first define $S$ to be the space of $\mathfrak{g}$ valued 1-forms on $P$ parameterised by elements of $G$

$$
S=\left\{s: G \times P \rightarrow T^{*} P \otimes_{\mathbb{R}} \mathfrak{g} \mid p \mapsto s_{p}^{g_{0}} \text { is a } \mathfrak{g} \text { valued 1-form } \forall g_{0} \in \mathcal{G}\right\}
$$

and then define

$$
\begin{aligned}
S_{\mathcal{N}}:= & \left\{\left(N_{\varepsilon}\right)_{\varepsilon} \in S^{I}: \forall K \subset \subset P, \forall \mathcal{D} \in \mathcal{P}\left(P, T^{*} P\right) \forall L \subset \subset G \forall m \in \mathbb{N}:\right. \\
& \left.\sup _{(p, g) \in K \times L}\left\|\mathcal{D} N_{\varepsilon, p}^{g}\right\|=O\left(\varepsilon^{m}\right)\right\} .
\end{aligned}
$$

Here \|\| denotes the norm induced on the fibres of $T^{*} P \otimes_{\mathbb{R}} \mathfrak{g}$ by any Riemannian metric on $P$ and any norm on $\mathfrak{g}$. Clearly $S_{\mathcal{N}}$ does not depend on these choices.

We are now in a position to give a definition of a generalised connection.

Definition 4.1 A generalised connection form $\omega$ on a principal fibre bundle $P(M, G)$ is an element of $\Omega_{\mathcal{G}}^{1}(P, \mathfrak{g})$ which satisfies the following conditions

(i) The map $\left(p \mapsto \omega_{p}\left(\sigma_{p}(\cdot)\right)\right)=\mathrm{id} \in \mathcal{G}(P, L(\mathfrak{g}, \mathfrak{g}))$. 
(ii) $\exists\left(\omega_{\varepsilon}\right)_{\varepsilon}$ with $\omega=\left[\left(\omega_{\varepsilon}\right)_{\varepsilon}\right] \exists N \in S_{\mathcal{N}}$ s.t. $\forall g \in G \forall V \in T_{R_{g} p} P$ :

$$
\omega_{R_{g} p, \varepsilon}(V)=\operatorname{ad}\left(g^{-1}\right) \omega_{p, \varepsilon}\left(R_{g *}^{-1} V\right)+N_{p, \varepsilon}^{g}(V) .
$$

Note that $\exists\left(\omega_{\varepsilon}\right)_{\varepsilon}$ and $\forall\left(\omega_{\varepsilon}\right)_{\varepsilon}$ is equivalent in (ii) above, i.e., every representative of $\omega$ satisfies the condition if one does. This is easily verified from the definitions of $\Omega_{\mathcal{G}}^{1}$ and $S_{\mathcal{N}}$.

In formulating this definition we began with the classical definition of a connection 1-form on $P$ and adapted the definition in the natural way to the space of generalised 1-forms on $P$. An alternative but less satisfactory approach would have been to work with 1-parameter families of connection 1-forms. We now show that although our definition is more general it allows one to locally choose representatives $\left(\omega_{\varepsilon}\right)_{\varepsilon}$ for $\omega$ such that each $\omega_{\varepsilon}$ is a connection 1-form.

Theorem 4.2 Let $\omega \in \Omega_{\mathcal{G}}^{1}(P, \mathfrak{g})$ be a generalised connection 1-form on $P(M, G)$ and let $U$ be a relatively compact open set $U \subset M$. Then there exist representatives $\left(\omega_{\varepsilon}\right)_{\varepsilon}$ of $\left.\omega\right|_{\pi^{-1}(U)}$ and an $\varepsilon_{0}>0$ such that $\omega_{\varepsilon}$ is a classical connection 1-form on $\pi^{-1}(U)$ for all $\varepsilon<\varepsilon_{0}$.

Proof. Let $f: U \rightarrow f(U) \subset P$ be a local section of $P$. Given some representative $\left(\omega_{\varepsilon}\right)_{\varepsilon}$ for $\omega \in \Omega_{\mathcal{G}}^{1}(P, \mathfrak{g})$ we use $\omega_{\varepsilon}$ to construct a connection 1-form $\tilde{\omega}_{\varepsilon}$ on $\pi^{-1}(U)$. We first define the connection form at points $f(x)$ on the section and then extend it to other points on the fibre using property (ii) of a classical connection.

Let $H_{f(x), \varepsilon}=\left\{X \in T_{f(x)} P: \omega_{f(x), \varepsilon}(X)=0\right\}$. Then since $\omega_{f(x), \varepsilon}\left(\sigma_{f(x)}(A)\right)=$ $A+N_{f(x), \varepsilon}(A)$ for all $A \in \mathfrak{g}$ where $N_{f(x), \varepsilon} \rightarrow 0$ as $\varepsilon \rightarrow 0$ we see that for sufficiently small $\varepsilon$ the linear map $\omega_{f(x), \varepsilon}: T_{f(x)} P \rightarrow \mathfrak{g}$ is onto. Hence for $\varepsilon$ sufficiently small, the kernel, $H_{f(x), \varepsilon}$, has dimension $\operatorname{dim} M$ and is transverse to the fibre at $f(x)$. Since $U$ is relatively compact we can find an $\varepsilon_{0}>0$ so that this is true for all $x \in U$ and all $\varepsilon<\varepsilon_{0}$.

We may now use $H_{f(x), \varepsilon}$ to uniquely write any vector $X \in T_{f(x)} P$ as $X=$ $X_{h \varepsilon}+X_{v \varepsilon}$ where $X_{h \varepsilon} \in H_{f(x), \varepsilon}$ and $X_{v \varepsilon} \in V_{f(x)}$. This enables us to define the connection form $\tilde{\omega}_{\varepsilon}$ at $f(x)$ (for $\varepsilon<\varepsilon_{0}$ ) by

$$
\tilde{\omega}_{f(x), \varepsilon}(X)=\sigma_{f(x)}^{-1}\left(X_{v, \varepsilon}\right) .
$$

In particular we see that if $X \in H_{f(x), \varepsilon}$ then $\tilde{\omega}_{f(x), \varepsilon}(X)=0$ and vice-versa, so that the horizontal subspaces of $\omega_{\varepsilon}$ and $\tilde{\omega}_{\varepsilon}$ agree at $f(x)$, i.e.,

$$
H_{f(x), \varepsilon}=\tilde{H}_{f(x), \varepsilon} .
$$

Also if $X \in V_{f(x)}$, then $\tilde{\omega}_{f(x), \varepsilon}(X)=\sigma_{f(x)}^{-1}(X)$ and hence

$$
\tilde{\omega}_{f(x), \varepsilon}\left(\sigma_{f(x)}(A)\right)=A, \forall A \in \mathfrak{g} .
$$

We now suppose that $p$ is a general point in $\pi^{-1}(U)$ and let $x=\pi(p)$. Then we may uniquely define $g(p) \in G$ by the requirement that $R_{g(p)} f(x)=p$. We 
now use the transformation property of a classical connection to define $\tilde{\omega}_{\varepsilon}$ at $p$ according to

$$
\tilde{\omega}_{p, \varepsilon}(W):=\operatorname{ad}\left(g(p)^{-1}\right) \tilde{\omega}_{f(x), \varepsilon}\left(R_{g(p) *}^{-1} W\right), \forall W \in T_{p} P
$$

Note in particular that if $W \in V_{p}$, then $R_{g(p) *}^{-1}(W) \in V_{f(x)}$ so that

$$
\begin{aligned}
\tilde{\omega}_{p, \varepsilon}(W) & =\operatorname{ad}\left(g(p)^{-1}\right) \sigma_{f(x)}^{-1}\left(R_{g(p) *}^{-1} W\right) \\
& =\operatorname{ad}\left(g(p)^{-1}\right) \operatorname{ad}(g(p)) \sigma_{p}^{-1}(W) \\
& =\sigma_{p}^{-1}(W)
\end{aligned}
$$

and hence condition (i) for a connection holds for $\tilde{\omega}_{\varepsilon}$ at all points $p \in \pi^{-1}(U)$. Since $g\left(R_{h} p\right)=g(p) h$, condition (ii) for a connection follows from

$$
\tilde{\omega}_{R_{h} p, \varepsilon}(V)=\operatorname{ad}\left(h^{-1} g(p)^{-1}\right) \tilde{\omega}_{f(x), \varepsilon}\left(R_{g(p) *}^{-1} R_{h *}^{-1} V\right)=\operatorname{ad}\left(h^{-1}\right) \tilde{\omega}_{p, \varepsilon}\left(R_{h *}^{-1} V\right) .
$$

Finally, all the maps involved are smooth so that $\tilde{\omega}_{\varepsilon}$ is indeed a connection form on $\pi^{-1}(U) \subset P$ for each $\varepsilon<\varepsilon_{0}$.

We next show that $\omega_{\varepsilon}-\tilde{\omega}_{\varepsilon}$ is negligible, so that $\left(\tilde{\omega}_{\varepsilon}\right)_{\varepsilon}$ is also a representative for the generalised connection $\omega$.

Let $p \in \pi^{-1}(U)$. Since $\tilde{\omega}_{\varepsilon}$ is a connection we may write the tangent space at $P$ as the direct sum $T_{p} P=\tilde{H}_{p, \varepsilon} \oplus V_{p}$ where $\tilde{H}_{p, \varepsilon}$ is the horizontal subspace of $T_{p} P$ with respect to $\tilde{\omega}_{\varepsilon}$.

We first suppose that $X \in V_{p}$, then

$$
\omega_{p, \varepsilon}(X)=\sigma_{p}^{-1}(X)+N_{p, \varepsilon}\left(\sigma_{p}^{-1}(X)\right)
$$

and, on the other hand

$$
\tilde{\omega}_{p, \varepsilon}(X)=\sigma_{p}^{-1}(X)
$$

Hence if $X \in V_{p}$ then

$$
\omega_{p, \varepsilon}(X)-\tilde{\omega}_{p, \varepsilon}(X)=N_{p, \varepsilon}\left(\sigma_{p}^{-1}(X)\right) .
$$

Since $N$ is an element of $\mathcal{N}(P, L(\mathfrak{g}, \mathfrak{g}))$ and $\sigma$ is smooth, the difference $\omega_{p, \varepsilon}(X)-$ $\tilde{\omega}_{p, \varepsilon}(X)$ satisfies the required estimates for $p$ in the compact subset $K \subset \pi^{-1}(U)$, when $X \in V_{p}$, uniformly for $\|X\| \leqslant 1$ (with \|\| induced on $T_{p} P$ by any Riemannian metric on $P$ ).

We now suppose that $X \in \tilde{H}_{p, \varepsilon}$. We first note that this implies $R_{g(p) *}^{-1} X \in$ $\tilde{H}_{f(x), \varepsilon}=H_{f(x), \varepsilon}$ and thus $\omega_{f(x), \varepsilon}\left(R_{g(p) *}^{-1} X\right)=0$. Since $p=R_{g(p)} f(x)$ and $\omega$ is a generalised connection we may therefore write

$$
\begin{aligned}
\omega_{p, \varepsilon}(X) & =\omega_{R_{g(p)} f(x), \varepsilon}(X) \\
& =\operatorname{ad}\left(g(p)^{-1}\right) \omega_{f(x), \varepsilon}\left(R_{g(p) *}^{-1} X\right)+N_{p, \varepsilon}^{g(p)}(X) \\
& =N_{p, \varepsilon}^{g(p)}(X) .
\end{aligned}
$$


On the other hand

$$
\tilde{\omega}_{p, \varepsilon}(X)=0
$$

since $X \in \tilde{H}_{p, \varepsilon}$. So that

$$
\omega_{p, \varepsilon}(X)-\tilde{\omega}_{p, \varepsilon}(X)=N_{p, \varepsilon}^{g(p)}(X) .
$$

Because $p$ ranges over a compact set $K, g(p)$ also lies within a compact set and since $N \in S_{\mathcal{N}}$, the difference $\omega_{p, \varepsilon}(X)-\tilde{\omega}_{p, \varepsilon}(X)$ satisfies the required estimates when $X \in \tilde{H}_{p, \varepsilon}$ uniformly for $p$ varying in a compact set and uniformly for $\|X\| \leqslant 1$.

Using $\tilde{\omega}_{\varepsilon}$, any $X \in T_{p} P$ with $\|X\| \leqslant 1$ can be written as $X_{v}+X_{h \varepsilon}$ with $X_{v} \in V_{p}, X_{h \varepsilon} \in \tilde{H}_{p \varepsilon}$ and $\left\|X_{v}\right\|,\left\|X_{h \varepsilon}\right\| \leqslant 1$. Therefore the mapping norm of $\omega-\tilde{\omega}$ with respect to the norm induced on the fibres of $T P$ by any Riemannian metric on $P$ satisfies the $\Omega_{\mathcal{N}}^{1}\left(\pi^{-1}(U), \mathfrak{g}\right)$-estimates, i.e., $\omega=\tilde{\omega}$ in $\Omega_{\mathcal{G}}^{1}\left(\pi^{-1}(U), \mathfrak{g}\right)$, as claimed.

We now show that one may use a generalised connection to define a generalised projection from $T P$ to the space of vertical vectors.

Definition 4.3 Let $\omega$ be a generalised connection 1-form on $P$ and let $\left(\omega_{\varepsilon}\right)_{\varepsilon}$ be a representation for $\omega$. Then we define the family of vector bundle homomorphisms $\pi_{v, \varepsilon}: T P \rightarrow T P$ by

$$
\pi_{v, \varepsilon}(V)=\sigma_{p}\left(\omega_{p, \varepsilon}(V)\right), \quad \forall p \in P, \forall V \in T p P
$$

and set $\pi_{v}:=\left[\left(\pi_{v, \varepsilon}\right)_{\varepsilon}\right] \in \operatorname{Hom}_{\mathcal{G}}[T P, T P]$

Since $\sigma_{p}$ is a linear isomorphism which depends smoothly on $p$ and does not depend on $\varepsilon, \pi_{v}$ is well-defined. Furthermore by choosing a representation for $\omega$ for which each $\omega_{\varepsilon}$ is a connection (which is possible by the previous theorem) we see that if $V \in T_{p} P$ then $\pi_{\varepsilon, v}(V) \in V_{p}$ for all $\varepsilon$ so that $\pi_{v}$ defines a generalised projection onto the space of vertical vectors as claimed.

Definition 4.4 We define $\pi_{h} \in \operatorname{Hom}_{\mathcal{G}}[T P, T P]$, the projection onto the space of horizontal vectors, by $\pi_{h}(V)=i d_{T P}-\pi_{v}$.

Since $\pi_{v} \in \operatorname{Hom}_{i d_{X}}[T P, T P]$, this definition is justified by the remark following 5.8 in 24. Again by taking a representation for $\omega$ consisting of connections one sees from the corresponding classical result that $\omega\left(\pi_{h}(\cdot)\right)=0$ in $\Omega^{1}(P, \mathfrak{g})$. Moreover, for the distinguished representative given by the above theorem each $\pi_{h, \varepsilon}$ projects onto the kernel of $\omega_{\varepsilon}$.

\section{Generalised Curvature}

As we have seen at each point $p \in P$ a classical connection $\omega$ defines a projection $\pi_{h}: T_{p} P \rightarrow H_{p}$ onto the horizontal subspace at $p$. This enables one to define the exterior covariant derivative $D$ of a $\mathfrak{g}$ valued $\mathrm{r}$-form $\phi$ by

$$
D \phi\left(V_{1}, \ldots, V_{r+1}\right)=d \phi\left(\pi_{h} V_{1}, \ldots, \pi_{h} V_{r+1}\right)
$$


Since both $\pi_{h}$ and $d$ are well defined in the generalised case the above definition may be immediately extended to generalised forms.

Classically one applies the exterior covariant derivative to the connection form to define a 2 -form $\Omega=D \omega$ with values in $\mathfrak{g}$, which is called the curvature form of the connection. An explicit calculation then establishes the structure equation

$$
\Omega(U, V)=d \omega(U, V)+[\omega(U), \omega(V)]
$$

Note that this differs from the corresponding formula in [17] due to our choice of a different convention for exterior product and exterior derivative which are those of [4].

We use the above formula to define the curvature of a generalised connection.

Definition 5.1 Let $\omega \in \Omega_{\mathcal{G}}^{1}(P, \mathfrak{g})$ be a generalised curvature 1-form on $P(M, G)$. The generalised curvature two form $\Omega \in \Omega_{\mathcal{G}}^{2}(P, \mathfrak{g})$ is defined to be $\left[\left(\Omega_{\varepsilon}\right)\right]$ where

$$
\Omega_{p, \varepsilon}(U, V)=d \omega_{p, \varepsilon}(U, V)+\left[\omega_{p, \varepsilon}(U), \omega_{p, \varepsilon}(V)\right], \forall U, V \in T_{p} P
$$

An important feature of the classical curvature 2 -form is that it is equivariant under $R_{g}$ by the adjoint representation. We now show that this remains true for a generalised connection.

Theorem 5.2 Let $\Omega$ be the generalised curvature of a generalised connection $\omega$ then

$$
\left(R_{g}^{*} \Omega\right)(U, V)=\operatorname{ad}\left(g^{-1}\right) \Omega(U, V)
$$

where $R_{g}^{*}$ is the pull-back of the generalised 2-form $\Omega$ by the map $R_{g}: P \rightarrow P$ and is defined by $R_{g}^{*} \Omega:=\left[\left(R_{g}^{*} \Omega_{\varepsilon}\right)\right]$.

Note that this equation is to be interpreted in the same generalised sense as the corresponding equation for the connection. Namely, given a representative $\left(\Omega_{\varepsilon}\right)_{\varepsilon}$ for $\Omega$ and a fixed value of $g \in G$ then the representatives for the left and right hand sides differ by an element of $\Omega_{\mathcal{N}}^{2}(P, \mathfrak{g})$

Proof.

Let $U, V \in T_{p} P$ then

$$
\begin{aligned}
\left(R_{g}^{*} \Omega\right)_{p, \varepsilon}(U, V)= & \Omega_{R_{g} p, \varepsilon}\left(R_{g *} U, R_{g *} V\right) \\
= & (d \omega)_{R_{g} p, \varepsilon}\left(R_{g *} U, R_{g *} V\right)+\left[\omega_{R_{g} p, \varepsilon}\left(R_{g *} U\right), \omega_{R_{g} p, \varepsilon}\left(R_{g *} V\right)\right] \\
= & \left(R_{g}^{*}(d \omega)\right)_{p, \varepsilon}(U, V)+\left[\omega_{R_{g} p, \varepsilon}\left(R_{g *} U\right), \omega_{R_{g} p, \varepsilon}\left(R_{g *} V\right)\right] \\
= & \left(d\left(R_{g}^{*} \omega\right)\right)_{p, \varepsilon}(U, V)+\left[\omega_{R_{g} p, \varepsilon}\left(R_{g^{*}} U\right), \omega_{R_{g} p, \varepsilon}\left(R_{g *} V\right)\right] \\
= & \operatorname{ad}\left(g^{-1}\right)(d \omega)_{p, \varepsilon}(U, V)+d N_{p, \varepsilon}^{g}\left(R_{g *} U, R_{g *} V\right) \\
& +\left[\operatorname{ad}\left(g^{-1}\right) \omega_{p, \varepsilon}(U)+N_{p, \varepsilon}^{g}\left(R_{g *} U\right), \operatorname{ad}\left(g^{-1}\right) \omega_{p, \varepsilon}(V)+N_{p, \varepsilon}^{g}\left(R_{g *} V\right)\right] \\
= & \operatorname{ad}\left(g^{-1}\right)\left\{d \omega_{p, \varepsilon}(U, V)+\left[\omega_{p, \varepsilon}(U), \omega_{p, \varepsilon}(V)\right]\right\} \\
& +d N_{p, \varepsilon}^{g}\left(R_{g *} U, R_{g *} V\right)+\left[N_{p, \varepsilon}^{g}\left(R_{g *} U\right), \operatorname{ad}\left(g^{-1}\right) \omega_{p, \varepsilon}(V)\right] \\
& +\left[\operatorname{ad}\left(g^{-1}\right) \omega_{p, \varepsilon}(U), N_{p, \varepsilon}^{g}\left(R_{g *} V\right)\right] \\
= & \operatorname{ad}\left(g^{-1}\right) \Omega_{p, \varepsilon}(U, V)+M_{p, \varepsilon}^{g}(U, V),
\end{aligned}
$$


where for fixed $g \in G, M^{g}(U, V) \in \Omega_{\mathcal{N}}^{2}(P, \mathfrak{g})$ since for fixed $g, N^{g} \in \Omega_{\mathcal{N}}^{1}(P, \mathfrak{g})$ which is a differential ideal.

The second key property of the curvature $\Omega$ is that it is a horizontal form. That is $U \in V_{p} \Rightarrow \Omega_{p}(U, V)=0, \forall V \in T_{p} P$. This follows immediately from the definition using the exterior covariant derivative but is less obvious from the definition using the structure equation. We now show that this result remains true in the generalised case. Note that even in the generalised case the definition of the vertical subspace $V_{p}$ does not depend upon $\varepsilon$ since it is defined using $\pi: P \rightarrow M$ and does not depend upon the generalised connection, (of course this is not true for the horizontal subspace).

Theorem 5.3 Let $\Omega \in \Omega_{\mathcal{G}}^{2}(P, \mathfrak{g})$ be a generalised 2-form, then $\Omega$ is a horizontal form.

Proof. By theorem 4.2 we may take local representatives $\omega_{\varepsilon}$ of the generalised connection $\omega$ which are themselves connection forms. The result then follows from the classical result.

The significance of the above two results is that the curvature is a tensorial 2 -form of type ad, (unlike the connection which is only pseudo-tensorial). This means that the pull-back of $\Omega$ onto $M$ by a local section transforms under the adjoint action on a change of section and does not have any inhomogeneous terms (unlike the connection $\omega$ ). This will be important in section 8 where we define characteristic classes for generalised connections. Another important result that we will use later is Bianchi's identity for the generalised curvature $\Omega$.

\section{Theorem 5.4 (Bianchi's identity) $D \Omega=0$}

Proof. By theorem 4.2 we may take local representatives $\omega_{\varepsilon}$ of the generalised connection $\omega$ which are themselves connection forms. The result then follows from taking the exterior derivative of the structure equation (25) and using the fact that by definition the connection vanishes on horizontal vectors.

\section{Horizontal Lifts and Holonomy}

Given a connection form $\omega$ on $P(M, G)$ we now show how this allows one to lift a curve $\gamma$ on the base manifold $M$ to a horizontal curve $\bar{\gamma}$ on the bundle $P$.

Definition 6.1 Let $\gamma:[a, b] \rightarrow M, t \mapsto \gamma(t)$ be a smooth curve on $M$. A horizontal lift of $\gamma$ is a curve $\bar{\gamma}:[a, b] \rightarrow P$ such that

(i) $\pi \circ \bar{\gamma}=\gamma$,

(ii) $\omega\left(\bar{\gamma}_{*}\left(\frac{\partial}{\partial t}\right)\right)=0$. 
Given some point $p_{0} \in \pi^{-1}(\gamma(a))$ there exists a unique lift $\bar{\gamma}$ such that $\bar{\gamma}(a)=p_{0}$ (Proposition 3.1 of Chapter II in [17]). We first choose a reference curve $f:[a, b] \rightarrow M$ satisfying $f(a)=p_{0}$ and $\pi \circ f=\gamma$, and look for a curve $g(t)$ in $G$ such that $\bar{\gamma}(t)=R_{g(t)} f(t)$ is horizontal. This is the case if the curve $g(t)$ in $G$ satisfies

$$
\dot{g}(t) g(t)^{-1}=-\omega\left(f_{*}\left(\frac{\partial}{\partial t}\right)\right) .
$$

Thus finding a horizontal lift for $\gamma$ which starts at $p_{0}$ is equivalent to obtaining a solution to (28) on $[a, b]$ which satisfies the initial condition $g(a)=e$. Since the right hand side is smooth and $[a, b]$ is compact there exists a unique solution to this equation, and hence there exists a unique lift $\bar{\gamma}$ starting from $p_{0}$. Note that if one chooses a different initial point $p_{1}=R_{g_{0}} p_{0}$ then the corresponding lift is $R_{g_{0}} \bar{\gamma}$.

The concept of horizontal lift is used to define the holonomy associated with a closed curve on $M$. Let $\gamma:[a, b] \rightarrow M$ be a closed curve with $\gamma(a)=$ $\gamma(b)=m$. If $\bar{\gamma}:[a, b] \rightarrow P$ is some horizontal lift of $\gamma$ then $\bar{\gamma}(a)$ and $\bar{\gamma}(b)$ will be points in the same fibre, so that they define an element $g$ of $G$ such that $\bar{\gamma}(b)=R_{g} \bar{\gamma}(a)$. We call $g$ the element of holonomy generated by $\bar{\gamma}$. Changing the starting point $\bar{\gamma}(a)$ by an element of $G$ simply translates the entire lift, so we see that the holonomy does not depend upon which horizontal lift is taken but is determined by $\gamma$. Furthermore the holonomy does not depend upon the particular parameterisation of $\gamma$, so the holonomy only depend upon the loop and the connection $\omega$. If one traverses the loop in the opposite direction the holonomy generated is the inverse of $g$. Similarly if loops $\gamma_{1}$ and $\gamma_{2}$ are two loops based at $m$ which generate elements of holonomy $g_{1}$ and $g_{2}$ respectively then the loop consisting of $\gamma_{1}$ followed by $\gamma_{2}$ generates $g_{2} g_{1}$. This leads to the important notion of holonomy group which encodes considerable information about the curvature of $P$.

The above concepts can be extended to the generalised setting. We start with the concept of horizontal lift

Definition 6.2 Let $\gamma \in \mathcal{G}[[a, b], M]$ be a generalised curve in $M$. A generalised horizontal lift of $\gamma$ is a curve $\bar{\gamma} \in \mathcal{G}[[a, b], P]$ such that

(i) $\pi \circ \bar{\gamma}=\gamma$ in $\mathcal{G}[[a, b], M]$.

(ii) $\omega_{\bar{\gamma}(.)}\left(\bar{\gamma}_{*}\left(\frac{\partial}{\partial t}\right)\right)=0$ in $\mathcal{G}[[a, b], \mathfrak{g}]$.

We note that $\omega_{\bar{\gamma}}$ is an element of the hybrid space $\mathcal{G}^{h}\left([a, b], T^{*} P \otimes \mathfrak{g}\right)$, cf. [23], Def. 4.4 and Th. 4.5 (i). On the level of representatives, the above conditions translate into

(i) $\pi \circ \bar{\gamma}_{\varepsilon}=\gamma_{\varepsilon}+r_{\varepsilon}$, where $r_{\varepsilon} \in \mathcal{N}([a, b], M)$

(ii) $\omega_{\bar{\gamma}_{\varepsilon}(t)}\left(\bar{\gamma}_{*, \varepsilon}\left(\frac{\partial}{\partial t}\right)\right)=N_{\varepsilon}(t)$, where $\left(N_{\varepsilon}\right)_{\varepsilon} \in \mathcal{N}([a, b], \mathfrak{g})$. 
In order to show that generalised lifts exist we may adapt the classical proof and show that there exist generalised flows $g_{\varepsilon}(t)$ such that

$$
\dot{g}_{\varepsilon}(t) g_{\varepsilon}(t)^{-1}=-\omega_{\varepsilon}\left(f_{*}\left(\frac{\partial}{\partial t}\right)\right) \text { in } \mathcal{G} .
$$

Again this equation must be interpreted in the generalised sense described in [21].

These ideas will be developed further in a future paper. For the moment we simply remark that in order to describe the generalised geometry of fibre bundles one requires a theory which not only permits the multiplication of generalised functions (in order to define the curvature), but can also deal with generalised functions (such as $\bar{\gamma}$ ) valued in a manifold, the composition of generalised functions (such as $\omega \circ \bar{\gamma}_{*}$ ) and generalised flows on manifolds (such as the equation for $g(t))$ all of which are available in the Colombeau theory as described in [22], [23], 19], 24] and 21].

\section{Generalised Connections in Vector Bundles and Covariant Derivatives}

In many applications one is interested in a connection or covariant derivative defined on an associated vector bundle rather than a connection on a principal bundle. For example in gauge theory the matter fields or Higgs fields are defined on associated bundles and in general relativity the connection used is defined on the tangent bundle $T M$, which may be regarded as an associated bundle of the frame bundle $L M$. We start by defining a covariant derivative (or a connection) on a vector bundle $E$.

A covariant derivative on a vector bundle $E$ is a map

$$
\begin{aligned}
\mathfrak{X}(M) \times \Gamma(M, E) & \rightarrow \Gamma(M, E) \\
(X, V) & \mapsto \nabla_{X} V
\end{aligned}
$$

which satisfies the conditions

(1) $\nabla_{X} V$ is $\mathbb{R}$-linear in $V$,

(2) $\nabla_{X} V$ is $C^{\infty}(M)$-linear in $X$,

(3) $\nabla_{X}(\lambda V)=\lambda \nabla_{X} V+X(\lambda) \cdot V, \quad \forall \lambda \in C^{\infty}(M)$.

We now briefly consider the way in which a connection on a principal bundle may be used to define a covariant derivative on an associated vector bundle and show how this construction may be extended to the case of a generalised connection.

We begin by reviewing the construction of an associated vector bundle. Let $P(M, G)$ be a principal fibre bundle and let $\rho: G \rightarrow G L(n, \mathbb{R}), g \mapsto \rho_{g}$ be a 
representation of $G$ on $\mathbb{R}^{n}$. Then on the product manifold $P \times \mathbb{R}^{n}$ we may define a right action of $G$ according to

$$
\begin{aligned}
G \times\left(P \times \mathbb{R}^{n}\right) & \rightarrow P \times \mathbb{R}^{n} \\
(g,(u, \xi)) & \mapsto\left(R_{g} u, \rho_{g}^{-1} \xi\right)
\end{aligned}
$$

Now let $E=P \times_{G} \mathbb{R}^{n}$ be the quotient space under this action and let $u \xi \in E$ represent the equivalence class of $(u, \xi) \in P \times \mathbb{R}^{n}$. The projection which takes $(u, \xi) \in P \times G \mapsto \pi(u) \in M$ induces a projection $\pi_{E}: E \rightarrow M$. Every point $x \in$ $M$ has a neighbourhood $U$ such that $\pi^{-1}(U)$ is isomorphic to $U \times G$. Indeed let $\Phi: \pi^{-1}(U) \rightarrow U \times G$ be such a local trivialisation then we can use this to define a local trivialisation of $E$ through a map $\Psi: \pi^{-1}(U) \rightarrow U \times \mathbb{R}^{n}$ which takes $u \xi$ to $\rho\left(\Phi_{2}(u)\right) \xi$. Note that this does not depend upon the choice of representation of the element of $E$ since an equivalent representation $\left(R_{h} u\right)\left(\rho_{h}^{-1} \xi\right)$ gets mapped to the same point. We now introduce a differentiable structure on $E$ by requiring that $\pi_{E}^{-1}(U)$ is actually an open submanifold and $\Psi$ is a diffeomorphism. We then say that $E\left(M, \mathbb{R}^{n}, G, P, \rho\right)$ is a vector bundle associated to the principal bundle $P(M, G)$.

We next briefly recall how a (classical) connection on $P$ may be used to define a covariant derivative on $E$. Let $\Phi: \pi^{-1}(U) \rightarrow U \times G$ and $\Psi: \pi_{E}^{-1}(U) \rightarrow$ $U \times \mathbb{R}^{n}$ be the local trivialisations described above. Let $\gamma$ be a curve connecting $\gamma(0)=x_{0}$ and $\gamma(1)=x_{1}$ in $U$ and $\bar{\gamma}$ be a horizontal lift of the curve to $P$. Then we may use $\gamma$ to define an isomorphism $\tau_{1}^{0}$ between $\pi_{E}^{-1}\left(x_{0}\right)$ and $\pi_{E}^{-1}\left(x_{1}\right)$. Let $V_{0} \in \pi_{E}^{-1}\left(x_{0}\right)$ then we define $V_{1}=\tau_{1}^{0}\left(V_{0}\right)$ by requiring that

$$
\xi_{1}=\rho\left(g_{1} g_{0}^{-1}\right) \xi_{0}
$$

where $\Psi\left(V_{i}\right)=\left(x_{i}, \xi_{i}\right)$ and $\Phi(\bar{\gamma}(i))=\left(x_{i}, g_{i}\right)$ for $i=1,2$.

It is a classical result that $V_{1}$ does not depend upon either the choice of trivialisation or upon the particular lift $\bar{\gamma}$ chosen but only on the connection on $P$ and the curve $\gamma$. More generally given a curve $\gamma$ defined on some interval $J$ we may define $\tau_{t}^{s}(V)$ to be the result of parallely propagating the vector $V$ from $\gamma(s)$ to $\gamma(t)$. We use this to define the covariant derivative of a field $V(x) \in \Gamma(M, E)$ in the direction of the tangent to the curve at the point $\gamma(0)$ as

$$
\nabla_{\dot{\gamma}(0)} V(\gamma(0))=\lim _{h \rightarrow 0} \frac{1}{h}\left[\tau_{0}^{h}(V(\gamma(h)))-V(\gamma(0))\right]
$$

It is not hard to see that this only depends upon the direction of the tangent $X=\dot{\gamma}(0)$ and not the curve $\gamma$ so we may use the above to define $\nabla_{X} V$ at $\gamma(0)$. By using this formula at every point $x$ we may define the covariant derivative of the field $V \in \Gamma(M, E)$ with respect to the vector field $X \in \mathfrak{X}(M)$. It is readily verified that the covariant derivative defined in this way satisfies properties (1)$(3)$.

Now let $\left(V_{\alpha}, \Psi_{\alpha}\right)$ be a vector bundle chart for $E$ with coordinates $x^{i}, i=$ $1, \ldots, m$ on $M$ and let $e_{A}, A=1, \ldots n$ be the fields on $E$ induced by the 
canonical basis on $\mathbb{R}^{n}$. Then we may define the $m n^{2}$ functions $\Gamma_{i A}^{B}$ (which are the coefficients of the connection on $E$ ) by

$$
\nabla_{\partial_{i}} e_{A}=\Gamma_{i A}^{B} e_{B}
$$

An explicit formula for the connection coefficients in terms of the connection 1-form on $P$ may also be given. Let $x_{0} \in V_{\alpha}$ and let $\Phi: \pi^{-1}(U) \rightarrow U \times G$ be some local trivialisation of $P$. Choose $p_{0} \in \pi^{-1}\left(x_{0}\right)$ so that $\Phi\left(p_{0}\right)=\left(x_{0}, e\right)$ and define the section $s: V_{\alpha} \rightarrow \pi^{-1}\left(V_{\alpha}\right)$ by $s(x)=\Phi^{-1}(x, e)$; then at $x=x_{0}$ the connection coefficients $\Gamma_{i A}^{B}$ are given by

$$
\Gamma_{i A}^{B} e_{B}=\left(\left(D_{e} \rho\right)\left(s^{*} \omega\left(\partial_{i}\right)\right)\right)\left(e_{A}\right)
$$

The covariant derivative of a field $V$ is then given by

$$
\nabla_{X} V=\left(X^{i} V_{, i}^{B}+\Gamma_{i A}^{B} V^{A} X^{i}\right) e_{B}
$$

It is not hard to show that a connection defined by (33) and (34) does not depend upon the choice of vector bundle chart and we note that the form of (34) automatically ensures that the covariant derivative satisfies properties (1)(3) above.

In the special case where the principal bundle $P(M, G)$ is the frame bundle $L M$ then the structure group $G$ is $G L(n, \mathbb{R})$. The tangent bundle is obtained as an associated bundle simply by taking the canonical representation of $G L(n, \mathbb{R})$ on $\mathbb{R}^{n}$ so that $\rho=\mathrm{id}$, and a local trivialisation of $T M$ is given by a system of local coordinates $x^{i}$ on $M$. A linear connection on the frame bundle then gives a covariant derivative defined by

$$
\nabla_{X} V=\left(X^{i} \partial_{i} V^{j}+\Gamma_{k i}^{j} V^{i} X^{k}\right) \partial_{j}
$$

where the connection coefficients are given by

$$
\nabla_{\partial_{i}} \partial_{j}=\Gamma_{i j}^{k} \partial_{k}
$$

We now extend these ideas to the generalised context. A generalised field $V$ is an element of the $\mathcal{G}$-module of generalised sections $\Gamma_{\mathcal{G}}(M, E)$. We first extend the definition of generalised linear connection on a manifold given in 23] to the case of a generalised connection on a vector bundle $E$.

Definition 7.1 (i) A generalised vector bundle connection $\hat{\nabla}$ on a vector bundle $E$ is a map

$$
\begin{aligned}
\mathcal{G}_{0}^{1}(M) \times \Gamma_{\mathcal{G}}(M, E) & \rightarrow \Gamma_{\mathcal{G}}(M, E) \\
(X, V) & \mapsto \hat{\nabla}_{X} V
\end{aligned}
$$

satisfying the following conditions 
(1) $\hat{\nabla}_{X} V$ is $\mathcal{R}$-linear in $V$,

(2) $\hat{\nabla}_{X} V$ is $\mathcal{G}(M)$-linear in $X$,

(3) $\hat{\nabla}_{X}(\lambda V)=\lambda \hat{\nabla}_{X} V+X(\lambda) \cdot V, \quad \forall \lambda \in \mathcal{G}(M)$.

(ii) Let $\left(V_{\alpha}, \Psi_{\alpha}\right)$ be a vector bundle chart for $E$ with coordinates $x^{i}, i=$ $1, \ldots, m$ on $M$ and $E_{A} A=1, \ldots n$ the fields on $E$ induced by the canonical basis on $\mathbb{R}^{n}$. We define the generalised connection coefficients for this chart to be the $m n^{2}$ functions $\hat{\Gamma}_{i A}^{B} \in \mathcal{G}\left(V_{\alpha}\right)$ given by

$$
\hat{\nabla}_{\partial_{i}} e_{A}=\hat{\Gamma}_{i A}^{B} e_{B}, \quad 1 \leqslant i \leqslant m, \quad 1 \leqslant A, B \leqslant m .
$$

Since $C^{\infty}(M)$ is a submodule of $\mathcal{G}(M)$ and the sheaf $\mathcal{G}(M)$ is fine (2) and (3) imply the localisability of any generalised vector bundle connection with respect to its arguments. (cf. [23]).

We now show how a generalised connection on $P(M, G)$ may be used to define a generalised vector bundle connection $\hat{\nabla}$ on $E$. The key point is that because of Theorem 3.2 given a connection $\omega \in \Omega_{\mathcal{G}}^{1}(P, \mathfrak{g})$ we may (locally) take representatives $\left(\omega_{\varepsilon}\right)_{\varepsilon}$ of $\omega$ which are themselves connections. Given a bundle chart $\left(V_{\alpha}, \Psi_{\alpha}\right)$ for $E$ we may as before define the one parameter family of functions

$$
\nabla_{\varepsilon, \partial_{i}} e_{A}=\Gamma_{\varepsilon, i A}^{B} e_{B}
$$

It is a straightforward computation to show using (33) that $\hat{\Gamma}_{A i}^{B}=\left(\Gamma_{\varepsilon, i A}^{B}\right)_{\varepsilon}$ defines an element of $\mathcal{G}\left(V_{\alpha}\right)$.

If we now define

$$
\nabla_{\varepsilon, X_{\varepsilon}} V_{\varepsilon}=\left(X_{\varepsilon}^{i} \partial_{i} V_{\varepsilon}^{A}+\Gamma_{\varepsilon, i B}^{A} V_{\varepsilon}^{B} X_{\varepsilon}^{i}\right) e_{A}
$$

then this defines a generalised vector bundle connection by setting $\hat{\nabla}_{X} V=$ $\left[\left(\nabla_{\varepsilon, X_{\varepsilon}} V_{\varepsilon}\right)_{\varepsilon}\right]$. Furthermore the form of equation (39) guarantees that the generalised covariant derivative satisfies conditions (1)-(3).

As remarked earlier an important example of a connection on an associated bundle is provided by a linear connection on $T M$ regarded as an associated bundle of $L M$. In this case local coordinates $x^{i}$ on $M$ provide a local section $s: M \rightarrow L M$ by associating with $x \in U \subset M$ the frame $\left\{\partial_{i}\right\}_{i=1}^{n}$. If $\omega_{\varepsilon}$ is a generalised connection 1-form on $L M$ with values in $g l(n, \mathbb{R})$ then we define the generalised Christoffel symbols $\Gamma_{j k, \varepsilon}^{i}$ by

$$
s^{*} \omega_{\varepsilon}(V)=\Gamma_{j k, \varepsilon}^{i} V^{j} E_{i}^{k}
$$

where $X=X^{i} \partial_{i}$ and $E_{j}^{i}$ is the basis for $g l(n, \mathbb{R})$ given by the matrix with a one in the $i$-the column and $j$-th row. Then in accordance with (39) this defines a generalised covariant derivative $\hat{\nabla}$ by

$$
\hat{\nabla}_{\varepsilon, X} V=\left(X^{i} \partial_{i} V^{j}+\Gamma_{\varepsilon, k i}^{j} V^{i} X^{k}\right) \partial_{j}
$$


which satisfies conditions (1)-(3) of Definition 7.1 Since $E=T M$ in this case these are precisely conditions D1-D3 of Definition 5.1 of 23. Hence a generalised connection form on $L M$ defines a generalised linear connection on $T M$ according to the definition of 23 .

\section{Characteristic Classes for Generalised Con- nections}

The curvature form $\Omega$ of a connection on a principal bundle $P$ together with an invariant polynomial $f$ may be used to construct a closed 2-form $\bar{f}(\Omega)$ on the base $M$. It turns out that the element of the de Rham cohomology $H^{*}(M)$ associated with $\bar{f}(\Omega)$ does not depend upon the choice of connection form $\omega$ on $P$ and is a topological invariant known as a characteristic class. For the case of a connection on a vector bundle with fibre $V$ one can view the bundle as an associated bundle of a principal $G L(V)$ bundle and calculate the characteristic classes of this. An important example of this are the Chern classes whose construction we describe below.

We start by showing how to construct closed forms on $M$. Let $f$ be a $k$-linear symmetric map from $\mathfrak{g}$ to $\mathbb{R}$ which is ad-invariant, so that

$$
f\left(\operatorname{ad} g V_{1}, \ldots, \operatorname{ad} g V_{k}\right)=f\left(V_{1}, \ldots, V_{k}\right), \quad \forall g \in G, \quad \forall V_{i} \in \mathfrak{g}
$$

We now define the $2 k$-form $f(\Omega)$ on $P$ by

$$
f(\Omega)\left(v_{1}, \ldots, v_{2 k}\right)=\frac{1}{(2 k) !} \sum_{\sigma} \operatorname{sign} \sigma f\left(\Omega\left(v_{\sigma(1)}, v_{\sigma(2)}\right), \ldots, \Omega\left(v_{\sigma(2 k-1)}, v_{\sigma(2 k)}\right)\right)
$$

where $v_{i} \in T_{p} P$ and the sum is over all permutations $\sigma$.

We may use $f(\Omega)$ to define a $2 k$-form $\bar{f}(\Omega)$ on $M$ as follows. Let $x \in M$ and $u_{i} \in T_{x} M$. Now let $p \in P$ be such that $\pi(p)=x$ and let $v_{i} \in T_{p} P$ be such that $D \pi_{p}\left(v_{i}\right)=u_{i}$, then we define

$$
\bar{f}(\Omega)_{x}\left(u_{1}, \ldots, u_{2 k}\right)=f(\Omega)_{p}\left(v_{1}, \ldots, v_{2 k}\right) .
$$

We note that the RHS does not depend upon the choice of $v_{i}$ which projects onto $u_{i}$ since any two such vectors differ by a vertical vector and $\Omega$ vanishes on vertical vectors, nor does the RHS depend upon the choice of $p$ since any two such points $p_{1}$ and $p_{2}$ are related by $p_{2}=R_{g} p_{1}$ for some $\mathfrak{g} \in G$ and $R_{g}^{*} \Omega=\operatorname{ad}\left(g^{-1}\right) \Omega$. Thus $f(\Omega)$ projects onto a unique $2 k$-form $\bar{f}(\Omega)$ on $M$.

We next observe that $\bar{f}(\Omega)$ is closed. This follows from the fact that $d(f(\Omega))=$ $D(f(\Omega))$ since $\Omega$ vanishes on horizontal vectors and $D(f(\Omega))=0$ by the Bianchi identity.

Finally using a homotopy argument one can show that given two connection 1 -forms on $P, \omega_{0}$ and $\omega_{1}$ then

$$
\bar{f}\left(\Omega_{0}\right)-\bar{f}\left(\Omega_{1}\right)=d Q
$$


where $Q$ depends upon both $\omega_{0}$ and $\omega_{1}$. Hence $\bar{f}\left(\Omega_{0}\right)$ and $\bar{f}\left(\Omega_{1}\right)$ represent the same cohomology class, a so-called characteristic class (see [4 for details).

The results of section 5 show that the above constructions may be extended to the case of a generalised connection.

Theorem 8.1 Let $\omega \in \Omega_{\mathcal{G}}^{1}(P, \mathfrak{g})$ be a generalised connection 1-form on $P$ with generalised curvature $\Omega$ and $f$ an ad-invariant $k$-multilinear map as above, then $f(\Omega)$ projects onto a unique closed $2 k$-form $\bar{f}(\Omega) \in \Omega_{\mathcal{G}}^{2 k}(M)$ which satisfies

$$
\pi^{*} \bar{f}(\Omega)=f(\Omega) \text {. }
$$

Proof. We define $f(\Omega)$ using equation (43). Then by Theorem [5.3] and Theorem $5.2 f(\Omega)$ is an invariant horizontal generalised form so that it projects onto a unique generalised $2 k$-form on $M$ given by equation (44).

The theory of generalised exterior calculus on a manifold is outlined in 22] where it is shown that all the classical operations may be extended to the generalised case. Using these ideas we may define the concept of the generalised de Rham cohomology of $M$. The following result clarifies the relationship between generalized and smooth de Rham cohomology.

Proposition 8.2 For each $p \geqslant 0$, the following isomorphism of real vector spaces holds:

$$
H_{\mathcal{G}}^{p}(M) \cong \mathcal{R} \otimes_{\mathbb{R}} H^{p}(M)
$$

Proof. For clarity, in this proof we denote by $d$ the usual exterior derivative on smooth forms and by $d^{\prime}$ the corresponding map on Colombeau forms. $H_{\mathcal{G}}^{*}$ is calculated through the following fine resolution of the sheaf of locally constant Colombeau functions:

$$
0 \longrightarrow \operatorname{ker}\left(d^{\prime}\right) \stackrel{d^{\prime}}{\longrightarrow} \Omega_{\mathcal{G}}^{0}(M) \stackrel{d^{\prime}}{\longrightarrow} \Omega_{\mathcal{G}}^{1}(M) \stackrel{d^{\prime}}{\longrightarrow} \ldots
$$

Consider now the vector spaces $\mathcal{R} \otimes_{\mathbb{R}} \Omega^{p}(M)$. Then for $p=0$, the kernel of $i d \otimes d: \mathcal{R} \otimes_{\mathbb{R}} C^{\infty}(M) \rightarrow \mathcal{R} \otimes_{\mathbb{R}} \Omega^{1}(M)$ is the set of locally constant Colombeau functions on $M$, i.e., ker $d^{\prime}$. Moreover, for $p \geqslant 1$ the map $\sum r_{i} \otimes \omega_{i} \mapsto \sum r_{i} \otimes\left[\omega_{i}\right]$ induces a bijection

$$
\operatorname{ker}(i d \otimes d) / \operatorname{Im}(i d \otimes d) \rightarrow \mathcal{R} \otimes_{\mathbb{R}} H^{p}(M)
$$

Thus we arrive at another fine resolution of ker $d^{\prime}$ of the form

$$
0 \longrightarrow \operatorname{ker}\left(d^{\prime}\right) \stackrel{i d \otimes d}{\longrightarrow} \mathcal{R} \otimes_{\mathbb{R}} C^{\infty}(M, \mathbb{R}) \stackrel{i d \otimes d}{\longrightarrow} \mathcal{R} \otimes_{\mathbb{R}} \Omega^{1}(M) \stackrel{i d \otimes d}{\longrightarrow} \ldots
$$

which, by the abstract de Rham theorem (31], ch. 5), calculates the same cohomology as above. The claim therefore follows from (47).

Definition 8.3 Let $Z_{\mathcal{G}}^{p}(M)$ be the $\mathcal{R}$-module of closed generalised $p$-forms

$$
Z_{\mathcal{G}}^{p}(M)=\left\{A \in \Lambda_{\mathcal{G}}^{p}(M) \mid d A=0\right\},
$$


and let $B_{\mathcal{G}}^{p}(M)$ be the $\mathcal{R}$-module of exact generalised $p$-forms

$$
B_{\mathcal{G}}^{p}(M)=\left\{A \in \Lambda_{\mathcal{G}}^{p}(M) \mid \exists B \in \Lambda_{\mathcal{G}}^{p-1}(M) \text { s.t. } A=d B\right\},
$$

then we define the $p$-th generalised de Rham cohomology module $H_{\mathcal{G}}^{p}(M)$ to be given by the quotient

$$
H_{\mathcal{G}}^{p}(M)=Z_{\mathcal{G}}^{p}(M) / B_{\mathcal{G}}^{p}(M)
$$

We now have the following Theorem

Theorem 8.4 Let $\omega \in \Omega_{\mathcal{G}}^{1}(P, \mathfrak{g})$ be a generalised connection 1-form on $P$ with generalised curvature $\Omega$ and $f$ an ad-invariant $k$-multilinear map as above, then $f(\Omega)$ projects onto a unique generalised closed $2 k$-form $\bar{f}(\Omega)$ which defines an element of $H_{\mathcal{G}}^{2 k}(M)$ which does not depend upon $\omega$.

Proof. By Theorem 8.1 above we know that $\bar{f}(\Omega)$ defines an element of $Z_{\mathcal{G}}^{2 k}(M)$. By Theorem 4.2 we may take a local representative $\left(\omega_{\varepsilon}\right)_{\varepsilon}$ of $\omega$ such that all $\omega_{\varepsilon}$ are connections. Using this and the classical result we see that given two different connections $\omega_{0}$ and $\omega_{1}$ then $\bar{f}\left(\Omega_{0}\right)-\bar{f}\left(\Omega_{1}\right)$ defines an element of $B_{\mathcal{G}}^{2 k}(M)$. Hence $\bar{f}(\Omega)$ defines an element of $H_{\mathcal{G}}^{2 k}(M)$ which does not depend upon the choice of generalised connection.

Since a (classical) characteristic class defines an element of the de Rham cohomology $H^{*}(M)$ which is independent of the connection one may integrate it over a cycle to obtain a number which does not depend upon the connection. In particular one can integrate a characteristic class in $H^{m}(M)$ over the whole manifold to obtain a topological invariant. A well known example of this is the Euler number of an even dimensional manifold which is the integral of the Euler class $\gamma$ of the frame bundle over $M$. For a two dimensional manifold the Euler class is proportional to the curvature two form and one obtains

$$
\chi(M)=\frac{1}{2 \pi} \int_{M} \Omega
$$

However if one is looking at a manifold with boundary this formula needs to be corrected by adding the integral of the geodesic curvature 1-form $\kappa_{g}$ over $\partial M$,

$$
\chi(M)=\frac{1}{2 \pi} \int_{M} \Omega+\frac{1}{2 \pi} \int_{\partial M} \kappa_{g}
$$

This extra boundary term is an example of a Chern-Simons term which has to be included when integrating over a manifold with boundary since by (45) two different connections induce characteristic classes which differ by an exact form $d Q$. By including a boundary integral corresponding to $Q$ one obtains an expression which does not depend upon the connection.

By Theorem 8.4 it is clear that these ideas may be extended to the generalised case to give an expression for the Euler number in terms of the integral of the generalised curvature, however in this case one should note that the integral of a generalised form is $\mathcal{R}$-valued rather than $\mathbb{R}$-valued. In fact in the generalised 
case it is more useful to turn formula (52) round to given an expression for the generalised curvature over a singular region in terms of the Euler number and a regular integral over the boundary

$$
\int_{M} \Omega=2 \pi \chi(M)-\int_{\partial M} \kappa_{g} .
$$

This method was used in [5 to show that the generalised curvature of a cone is associated to a delta function.

In the next section a similar idea will be used to relate monopole charges of generalised connections with $U(1)$ Chern classes and generalised instanton numbers with $S U(2)$ Chern classes. We therefore briefly review the general construction of these classes. Let $E$ be a complex vector bundle on a real manifold $M$ with typical fibre $\mathbb{C}^{n}$. Then such a bundle may be regarded as an associated bundle of the principal fibre bundle $P(M, G L(n, \mathbb{C}))$ and any connection on $E$ may be obtained as the induced connection of a suitable connection on $P$ (see [4] for details). One may construct invariant polynomials $f_{k} k=0, \ldots, n$ on $G L(n, \mathbb{C})$ by writing the characteristic polynomial of the matrix $A$ as

$$
\operatorname{det}(\lambda I-A / 2 \pi i)=\sum_{k=0}^{n} f_{n}(A) \lambda^{n-k}
$$

Now let $\omega$ be some generalised connection on $P$ then the generalised $k$-th Chern class of the vector bundle $E$ is the generalised cohomology class of the unique closed $2 k$-form $c_{k}(\Omega)$ on $M$ which is defined by requiring that

$$
\pi^{*} c_{k}(\Omega)=f_{k}(\Omega) .
$$

For a two dimensional manifold the only Chern number one can obtain is given by

$$
C_{1}(E)=\int_{M} c_{1}(\Omega) .
$$

However for a four dimensional manifold it is possible to construct two Chern numbers

$$
\begin{aligned}
& C_{2}(E)=\int_{M} c_{2}(\Omega) \\
& C_{1}^{2}(E)=\int_{M} c_{1}(\Omega) \wedge c_{1}(\Omega)
\end{aligned}
$$

\section{Weakly Singular Solutions of Yang-Mills equa- tions}

Historically the first example of a weakly singular solution of the Yang-Mills equations is Dirac's description of the magnetic monopole [10]. In this paper 
Dirac describes an electromagnetic field with an electromagnetic 4-potential given in spherical polar coordinates by

$$
A=\alpha / 2(\cos \theta-1) d \phi
$$

which he notes is singular at the origin $r=0$ and also along the negative $z$-axis where $\theta=\pi$. However he claims that if $\alpha=n \in \mathbb{N}$ the singularity on the axis is physically unimportant. This is because the integral around a small loop $\gamma$ round the negative $z$-axis is then given by

$$
\int_{\gamma} A=2 n \pi
$$

so that the singularity has the effect of changing the phase of the field by an integer multiple of $2 \pi$ which does not effect the physical field. If one now computes the electromagnetic field $F=d A$ produced by $\mathrm{A}$ in this case one finds that

$$
\begin{aligned}
F & =-n / 2 \sin \theta d \theta \wedge d \phi \\
& =\frac{n}{2 r^{3}}(x d z \wedge d y+y d x \wedge d z+z d y \wedge d x)
\end{aligned}
$$

which corresponds to an electric and magnetic field given by

$$
\mathbf{E}=0, \quad \text { and } \quad \mathbf{H}=\frac{n \mathbf{r}}{r^{3}}
$$

So that this solution has no electric field but a magnetic field which looks like an integer magnetic charge centred at the origin. Indeed if we integrate over the 2-sphere $S$ given by $t=$ const. and $x^{2}+y^{2}+z^{2}=R^{2}$ one finds

$$
\int_{S} \mathbf{H} \cdot d \mathbf{S}=4 \pi n
$$

On the other hand for a smooth vector field

$$
\int_{S} \mathbf{H} \cdot d \mathbf{S}=\int_{V} \nabla \cdot \mathbf{H} d v
$$

where $V$ is the interior of $S$. Now $\nabla \cdot\left(\frac{\mathbf{r}}{r^{3}}\right)=0$ for $\mathbf{r} \neq 0$, so that $\nabla . \mathbf{H}$ behaves like the Dirac delta function $4 \pi n \delta_{0}$ and the solution is said to describe a magnetic monopole. As Dirac pointed out the condition that the integral (58) is an integer multiple of $2 \pi$ leads to the quantisation of magnetic charge according to (60). However one is not justified in applying integral theorems in this case due to the singularities of $A$.

In modern treatments of the magnetic monopole one regards $i \mathbf{A}$ as giving a connection on a $U(1)$ bundle on $\mathbb{R}^{4} \backslash\{r=0\} \approx \mathbb{R}^{2} \times S^{2}$. One then avoids the 
singularity on the negative $z$-axis by giving different descriptions of the potential on $U_{+}=\mathbb{R}^{4} \backslash\{r=0$ or $z<0\}$ and on $U_{-}=\mathbb{R}^{4} \backslash\{r=0$ or $z>0\}$ according to

$$
\begin{array}{lll}
A=A_{+}=n / 2(\cos \theta-1) d \phi, & \text { on } & U_{+} \\
A=A_{-}=n / 2(\cos \theta+1) d \phi, & \text { on } & U_{-}
\end{array}
$$

Note that $i A_{+}$and $i A_{-}$are related by the gauge transformation $i A_{+}=i A_{-}-$ ind $\phi$ on the thin 'overlap' $U_{+} \cap U_{-}$and so define a $U(1)$ connection on $\mathbb{R}^{2} \times S^{2}$ and that both $A_{+}$and $A_{-}$agree with the field (59) on $U_{+} \cup U_{-}$. In terms of this description the magnetic charge associated with the monopole is then given in terms of the Chern class (see 4 for details).

However from the spacetime point of view there is nothing wrong at the origin and it would be preferable to work with a space with topology $\mathbb{R}^{4}$ rather than exclude the origin and work with a space of topology $\mathbb{R}^{2} \times S^{2}$. More importantly the above description relies upon the quantisation condition $\alpha \in \mathbb{N}$ to ensure that $A_{+}$and $A_{-}$define a $U(1)$ connection and this method can not be applied to the sort of weakly singular Yang-Mills connections we consider later which have fractional charges. We therefore give an alternative formulation of the Dirac monopole in terms of a singular Yang-Mills connection over $\mathbb{R}^{4}$.

Before doing so we consider the simpler case of a $U(1)$ connection which is given by

$$
A=i \alpha d \phi
$$

where $\alpha \in \mathbb{R}$. It is readily verified that away from the $z$-axis this connection is flat. However if one considers the holonomy generated by a small loop $\gamma$ encircling the axis one finds that it is given by $\exp (-2 \pi \alpha i)$ so that unless $\alpha \in \mathbb{Z}$ the loop generates a non-trivial element of holonomy. Furthermore if we consider the gauge equivalent connection given by

$$
\tilde{A}=A+g^{-1} d g
$$

where $g(\phi)=\exp (-i n \phi)$ (with $n \in \mathbb{Z})$, then $\tilde{A}$ generates the element of holonomy $\exp (-2 \pi(\alpha-n))$ and without loss of generality we may use such a change of gauge to ensure that $\alpha$ lies in the range $0 \leqslant \alpha<1$. If $\alpha=0$ then in this gauge the connection vanishes everywhere and is globally flat. If $\alpha \neq 0$ the holonomy is non-trivial and the connection cannot be extended to a regular connection on the whole of Minkowski space (including the $z$-axis). This is because in the simple $U(1)$ case the holonomy may be given in terms of the exponential of the integral of the curvature and so for a regular connection this tends to the identity as the area of the loop shrinks to zero.

Although it is not possible to give a description of (62) as a regular connection on the whole of Minkowski space it is possible to define a generalised connection on the whole of $\mathbb{R}^{4}$ which represents $A$. To do this we look at $A$ in Cartesian coordinates. In these coordinates $A$ is given by

$$
A=\frac{x d y-y d x}{x^{2}+y^{2}} i \alpha
$$


This is singular on the $z$-axis so we replace $A \in \Omega^{1}\left(\mathbb{R}^{4} \backslash \mathbb{R}^{2}\right)$ by a regular family of potentials $A_{\varepsilon}$ which represent an element in $\Omega_{\mathcal{G}}^{1}\left(\mathbb{R}^{4}, i \mathbb{R}\right)$

$$
A_{\varepsilon}=\frac{x d y-y d x}{x^{2}+y^{2}+\varepsilon^{2}} i \alpha
$$

The corresponding field $F_{\varepsilon}=d A_{\varepsilon}$ is then given by

$$
F_{\varepsilon}=\frac{2 i \varepsilon^{2} \alpha d x \wedge d y}{\left(x^{2}+y^{2}+\varepsilon^{2}\right)^{2}}
$$

If we now write this in cylindrical polar coordinates $(t, \rho, \phi, z)$ we find

$$
F_{\varepsilon}=\frac{2 i \varepsilon^{2} \alpha \rho d \rho \wedge d \phi}{\left(\rho^{2}+\varepsilon^{2}\right)^{2}}
$$

Then by undertaking a calculation very similar to that in [5] one can show that the generalised two form in $\Omega_{\mathcal{G}}^{2}\left(\mathbb{R}^{4}, i \mathbb{R}\right)$ represented by the family $\left(F_{\varepsilon}\right)_{\varepsilon}$ given by (67) is associated to the distributional 2-form $2 \pi i \alpha \delta^{(2)}(x, y) d x \wedge d y$, which corresponds to a magnetic field given in Cartesian coordinates by

$$
\mathbf{H}=4 \pi \alpha\left(0,0, \delta^{(2)}(x, y)\right)
$$

We now turn to the generalised description of the Dirac monopole. As in the previous example we start by looking at the Dirac potential $A$ given by (57) in Cartesian coordinates. In these coordinates $A$ is given by

$$
A=\frac{\alpha}{2}\left(\frac{z}{\left(x^{2}+y^{2}+z^{2}\right)^{1 / 2}}-1\right)\left(\frac{x d y-y d x}{x^{2}+y^{2}}\right)
$$

We first note that in these coordinates the coefficients of $d x$ and $d y$ both diverge as we approach the negative $z$-axis. To remedy this we again replace $A$ by a regular family of potentials $\left(A_{\varepsilon}\right)_{\varepsilon}$ which represent an element of $\Omega_{\mathcal{G}}^{1}\left(\mathbb{R}^{4}\right)$

$$
A_{\varepsilon}=\frac{\alpha}{2}\left(\frac{z}{\left(x^{2}+y^{2}+z^{2}+\varepsilon^{2}\right)^{1 / 2}}-1\right)\left(\frac{x d y-y d x}{x^{2}+y^{2}+\varepsilon^{2}}\right)
$$

The associated electromagnetic field is given by $F_{\varepsilon}=d A_{\varepsilon}$ and a direct calculation shows that

$$
F_{\varepsilon}=F_{\varepsilon}^{1}+F_{\varepsilon}^{2}+F_{\varepsilon}^{3}
$$

where

$$
\begin{aligned}
& F_{\varepsilon}^{1}=\frac{\alpha(x d z \wedge d y+y d x \wedge d z+z d y \wedge d x)}{2\left(r^{2}+\varepsilon^{2}\right)^{3 / 2}} \\
& F_{\varepsilon}^{2}=\frac{\alpha \varepsilon^{2} z d x \wedge d y}{2\left(r^{2}+\varepsilon^{2}\right)^{3 / 2}\left(x^{2}+y^{2}+\varepsilon^{2}\right)} \\
& F_{\varepsilon}^{3}=\left(\frac{z}{\left(r^{2}+\varepsilon^{2}\right)^{1 / 2}}-1\right) \frac{\alpha \varepsilon^{2} d x \wedge d y}{\left(x^{2}+y^{2}+\varepsilon^{2}\right)^{2}}
\end{aligned}
$$


The first term $F_{\varepsilon}^{1}$ gives the monopole expression (59) as $\varepsilon \rightarrow 0$ while the flux of the second term vanishes as $\varepsilon \rightarrow 0$. However the third term $F_{\varepsilon}^{3}$ diverges on the negative $z$-axis and its contribution must be taken into account in computing the flux integral. Now away from the negative $z$-axis $F_{\varepsilon}^{3} \rightarrow 0$ as $\varepsilon \rightarrow 0$, so that

$$
\begin{aligned}
\lim _{\varepsilon \rightarrow 0} \int_{S} F_{\varepsilon}^{3}= & \lim _{\varepsilon \rightarrow 0} \int_{S^{+}} F_{\varepsilon}^{3}+\lim _{\varepsilon \rightarrow 0} \int_{S^{-}} F_{\varepsilon}^{3} \\
= & 0-\lim _{\varepsilon \rightarrow 0} \int_{S^{-}} \frac{2 \alpha \varepsilon^{2} d x \wedge d y}{\left(x^{2}+y^{2}+\varepsilon^{2}\right)^{2}} \\
& +\lim _{\varepsilon \rightarrow 0} \int_{S^{-}}\left(1+\frac{z}{\left(r^{2}+\varepsilon^{2}\right)^{1 / 2}}\right) \frac{\alpha \varepsilon^{2} d x \wedge d y}{\left(x^{2}+y^{2}+\varepsilon^{2}\right)^{2}} \\
= & 0-\lim _{\varepsilon \rightarrow 0} \oint_{C} \alpha\left(\frac{x d y-y d x}{x^{2}+y^{2}+\varepsilon^{2}}\right)+0 \\
= & -\int_{0}^{2 \pi} \alpha d \phi \\
= & -2 \alpha \pi
\end{aligned}
$$

We therefore see that in the regularised description as well as the monopole term $F^{1}$ there is also another term $F^{3}$ which behaves like the flux due to a potential $\alpha d \phi$ which corresponds to a delta function like magnetic field along the negative $z$-axis. It is this term due to the 'wire singularity' that results in the Chern number vanishing (as it must do given the local trivialisation over $\mathbb{R}^{4}$ ) despite the presence of the monopole term. In terms of a $U(1)$ bundle description, if $\alpha=n \in \mathbb{Z}$, the connection ind $\phi$ generates a trivial holonomy $e^{2 m \pi i}=1$ when integrated round any closed curve $\gamma$ and it is this feature that enables one to give the usual modern description of a monopole in terms of a $U(1)$ bundle over $\mathbb{R}^{2} \times S^{2}$.

A more interesting example of a weakly singular connection is the "fractionally charged instanton" discovered by Forgacs et al [11] (see also [12, 3], 2]). This is a (Euclidean) self-dual $S U(N)$ Yang-Mills connection on the 4-sphere with a singularity along a 2-sphere. Rather than consider the exact instanton solutions we follow Råde [27] and look at the general class of weakly singular $S U(N)$ connections on the unit ball in Euclidean space. Furthermore for simplicity of exposition we will look in detail at the case $N=2$ since no new features arise for higher values of $N$.

Let $B$ denote the 4-ball $x^{2}+y^{2}+z^{2}+w^{2} \leqslant 1$ in Euclidean 4-space with $(x, y, z, w)$ Cartesian coordinates. Let $D$ denote the disk $z=0, w=0, x^{2}+y^{2} \leqslant$ 1. We may now define 4-dimensional cylindrical polar coordinates $(x, y, r, \phi)$ by $z=r \cos \phi$ and $w=r \sin \phi$. Let $P$ be an $S U(2)$ bundle over $B$, then by a singular $S U(2)$ connection on $P$ we mean a connection that in a local gauge may be written

$$
\omega=\tilde{\omega}+a
$$


where

$$
\tilde{\omega}=\left(\begin{array}{cc}
i \alpha & 0 \\
0 & -i \alpha
\end{array}\right) d \phi, \quad 2 \alpha \notin \mathbb{Z},
$$

and $a$ is a regular $s u(2)$-valued 1 -form. The first term defines a regular connection on $B \backslash D$, but the holonomy around a loop $\gamma$ round the disk $D$ is given by

$$
\left(\begin{array}{cc}
\exp (-2 \pi \alpha i) & 0 \\
0 & \exp (2 \pi \alpha i)
\end{array}\right)
$$

and it is the non-trivial nature of this holonomy that makes the connection singular on $D$. (Note the reason that we demand that $2 \alpha$ rather than $\alpha$ is not an integer is that in the $S U(2)$ case it is possible to regard $A$ as splitting as the direct sum of a trivial $S U(2)$ connection on $B$ and a flat $U(1)$ connection $2 i \alpha d \theta$ on $B \backslash D$ with holonomy $\exp (-4 \pi \alpha i)$ round $D$, see [27 for details).

More generally one can consider singular connections on 4-manifolds which have non-trivial limit holonomy along an embedded surface in the 4-manifold. A theorem by L Sibner and R Sibner [29] shows that a finite energy YangMills connection on the complement of an embedded surface in a 4-manifold has a well defined limit holonomy around the surface and that the connection can be extended across the surface if and only if the limit holonomy is trivial. Furthermore they also show that any such connection over $B \backslash D$ that is in $L^{2,1}$ locally and has curvature in $L^{p}$ globally is gauge equivalent to (72) (see also [28]) so there is no loss of generality in considering singular connections given by (172).

We now show how to replace (73) by a generalised connection on $B$ and hence also give a description of (72) as a generalised connection on $B$. This enables us to give an explicit formula for the curvature of the regularised version of (72). As usual we start by writing (73) in Cartesian coordinates

$$
\tilde{\omega}=\left(\begin{array}{cc}
i \alpha & 0 \\
0 & -i \alpha
\end{array}\right) \frac{x d y-y d x}{x^{2}+y^{2}}
$$

and introduce the corresponding regular family of connections according to

$$
\tilde{\omega}_{\varepsilon}=\left(\begin{array}{cc}
i \alpha & 0 \\
0 & -i \alpha
\end{array}\right) A_{\varepsilon}
$$

where $A_{\varepsilon}$ is the 1 -form given by

$$
A_{\varepsilon}=\frac{x d y-y d x}{x^{2}+y^{2}+\varepsilon^{2}}
$$

This may be used to define a generalised connection in $\Omega_{\mathcal{G}}^{1}(P, s u(2))$ which is represented by the family $\left(\omega_{\varepsilon}\right)_{\varepsilon}$ given in a local gauge by $\omega_{\varepsilon}=\tilde{\omega}_{\varepsilon}+a$. The corresponding curvature is an element of $\Omega_{\mathcal{G}}^{2}(P, s u(2))$ which is represented by the family given by

$$
F_{\varepsilon}=d \tilde{\omega}_{\varepsilon}+d a+\left[\tilde{\omega}_{\varepsilon}+a, \tilde{\omega}_{\varepsilon}+a\right]
$$


In order to calculate this we decompose the $s u(2)$ valued 1-form $a$ into its diagonal and off diagonal components

$$
\left(\begin{array}{cc}
a_{D} & a_{T} \\
-\bar{a}_{T} & -a_{D}
\end{array}\right)
$$

and use the fact that if $a$ and $b$ are two $s u(2)$-valued 1-forms then

$$
\begin{aligned}
& {[a, b]_{D}=-2 i \operatorname{Im}\left(a_{T} \wedge \bar{b}_{T}\right)} \\
& {[a, b]_{T}=2\left(a_{D} \wedge b_{T}-a_{T} \wedge b_{D}\right)}
\end{aligned}
$$

We then find that

$$
F_{\varepsilon}=F_{\varepsilon}^{1}+F^{2}
$$

where the first term is the singular part and is given in terms of its diagonal and off diagonal pieces by

$$
F_{\varepsilon, D}^{1}=d A_{\varepsilon}, \quad F_{\varepsilon, T}^{1}=-2 i \alpha a_{T} \wedge A_{\varepsilon}
$$

with $A_{\varepsilon}$ given by (77) and $d A_{\varepsilon}$ given by

$$
d A_{\varepsilon}=\frac{2 \varepsilon^{2} d x \wedge d y}{\left(x^{2}+y^{2}+\varepsilon^{2}\right)^{2}}
$$

while the second term is the regular part and is just the curvature of the regular part of the connection

$$
F^{2}=d a+[a, a]
$$

The curvature of the generalised connection therefore splits into a smooth part $F^{2} \in \Omega^{2}(P, s u(2))$ given by (83) and a singular part $F^{1} \in \Omega_{\mathcal{G}}^{2}(P, s u(2))$ represented by the family given by (81), the new feature in the $s u(2)$ case being the way that the off diagonal piece is the wedge product of the smooth and singular parts of the connection.

Acknowledgment: We would like to thank Andreas Cap for helpful discussions.

\section{References}

[1] M. F. Atiyah. Magnetic monopoles in hyperbolic spaces. In Vector bundles on algebraic varieties (Bombay, 1984), volume 11 of Tata Inst. Fund. Res. Stud. Math., pages 1-33. Bombay, 1987.

[2] P. J. Braam. Magnetic monopoles on three-manifolds. J. Differential Geom., 30 (1989) 425-464.

[3] A. Chakrabarti. Spherically and axially symmetric $\mathrm{SU}(N)$ instanton chains with monopole limits. Nuclear Phys. B, 248 (1984) 209-252. 
[4] Y. Choquet-Bruhat, C. DeWitt-Morette M. Dillard-Bleick. Analysis, Manifolds and Physics. North Holland, Amsterdam, 1978.

[5] C. J. S. Clarke, J. A. Vickers J. P. Wilson. Generalised functions and distributional curvature of cosmic strings. Class. Quant. Grav., 13 (1996) $2485-2498$.

[6] J. F. Colombeau. New Generalized Functions and Multiplication of Distributions. North Holland, Amsterdam, 1984.

[7] J. F. Colombeau. Elementary Introduction to New Generalized Functions. North Holland, Amsterdam, 1985.

[8] N. Dapić, M. Kunzinger S. Pilipović. Symmetry group analysis of weak solutions. Proc. London Math. Soc. (3), 84 (2002) 686-710.

[9] J. W. De Roever M. Damsma. Colombeau algebras on a $\mathcal{C}^{\infty}$-manifold. Indag. Mathem., N.S., 2 (1991).

[10] P. A. M. Dirac. Quantised singularities in the elctromagnetic field. Proc. Roy. Soc., A133 (1931) 60-72.

[11] P. Forgács, Z. Horváth L. Palla. Exact, fractionally charged self-dual solution. Phys. Rev. Lett., 46 (1981) 392-394.

[12] P. Forgács, Z. Horváth L. Palla. One can have noninteger topological charge. Z. Phys. C, 12 (1982) 359-360.

[13] M. Grosser, E. Farkas, M. Kunzinger R. Steinbauer. On the foundations of nonlinear generalized functions I, II. Mem. Amer. Math. Soc., 153 (2001).

[14] M. Grosser, M. Kunzinger, M. Oberguggenberger R. Steinbauer. Geometric Theory of Generalized Functions, volume 537 of Mathematics and its Applications 53\%. Kluwer Academic Publishers, Dordrecht, 2001.

[15] M. Grosser, M. Kunzinger, R. Steinbauer J. Vickers. A global theory of algebras of generalized functions. Adv. Math., 166 (2002) 179-206.

[16] F. Harvey H. B. Lawson. A theory of characteristic currents associated with a singular connection. Astérisque, 213 268, 1993.

[17] S. Kobayashi K. Nomizu. Foundations of differential geometry. Vol I. New York-London, 1963.

[18] A. Kriegl P. W. Michor. The Convenient Setting of Global Analysis, volume 53 of Math. Surveys Monogr. Amer. Math. Soc., Providence, RI, 1997.

[19] M. Kunzinger. Generalized functions valued in a smooth manifold. Monatsh. Math., 137 (2002) 31-49.

[20] M. Kunzinger M. Oberguggenberger. Group analysis of differential equations and generalized functions. SIAM J. Math. Anal., 31 (2000) 1192-1213. 
[21] M. Kunzinger, M. Oberguggenberger, R. Steinbauer J. A. Vickers. Generalized flows and singular ODEs on differentiable manifolds. Acta. Appl. Math., 80 (2003) 221-241. available electronically at http://arxiv.org/abs/math.FA/03040131

[22] M. Kunzinger R. Steinbauer. Foundations of a nonlinear distributional geometry. Acta Appl. Math., 71 (2002) 179-206.

[23] M. Kunzinger R. Steinbauer. Generalized pseudo-Riemannian geometry. Trans. Amer. Math. Soc., 354 (2002) 4179-4199.

[24] M. Kunzinger, R. Steinbauer J. A. Vickers. Intrinsic characterization of manifold-valued generalized functions. Proc. London Math. Soc., 87 (2003) 451-470.

[25] V. Marenich K. Packalen. On singular connections and geometrically atomic maps. Preprint math.DG/0301315, 2003.

[26] M. Oberguggenberger M. Kunzinger. Characterization of Colombeau generalized functions by their pointvalues. Math. Nachr., 203 (1999) 147-157.

[27] J. Råde. Singular Yang-Mills fields. Local theory. I. J. Reine Angew. Math., 452 (1994) 111-151.

[28] J. Råde. Singular Yang-Mills fields. II. J. Reine Angew. Math., 456 (1994) $197-219$.

[29] L. M. Sibner R. J. Sibner. Classification of singular Sobolev connections by their holonomy. Comm. Math. Phys., 144 (1992) 337-350.

[30] J. A. Vickers. Nonlinear generalized functions in general relativity. In Grosser, M., Hörmann, G., Kunzinger, M., Oberguggenberger, M., editor, Nonlinear Theory of Generalized Functions, volume 401 of Chapman $\&$ Hall/CRC Research Notes in Mathematics, pages 275-290, Boca Raton, 1999. CRC Press.

[31] F. W. Warner. Foundations of differentiable manifolds and Lie groups. Springer-Verlag, New York-Berlin, 1983. 